\title{
Wind-tunnel simulations of the suburban ABL and comparison with international standards
}

Article in Wind and Structures An International Journal · January 2011

DOI: 10.12989/was.2011.14.1.015

CITATIONS

22

1 author:

\section{Hrvoje Kozmar}

University of Zagreb

44 PUBLICATIONS 234 CITATIONS

SEE PROFILE
READS

130 


\title{
Wind-tunnel simulations of the suburban $\mathrm{ABL}$ and comparison with international standards
}

\author{
Hrvoje Kozmar* \\ Faculty of Mechanical Engineering and Naval Architecture, University of Zagreb, \\ Ivana Lucića 5, HR-10000 Zagreb, Croatia \\ (Received February 27, 2010, Accepted May 23, 2010)
}

\begin{abstract}
Three wind-tunnel simulations of the atmospheric boundary layer (ABL) flow in suburban country exposure were generated for length scale factors 1:400, 1:250 and 1:220 to investigate scale effects in wind-tunnel simulations of the suburban ABL, to address recommended wind characteristics for suburban exposures reported in international standards, and to test redesigned experimental hardware. Investigated parameters are mean velocity, turbulence intensity, turbulent Reynolds shear stress, integral length scale of turbulence and power spectral density of velocity fluctuations. Experimental results indicate it is possible to reproduce suburban natural winds in the wind tunnel at different length scales without significant influence of the simulation length scale on airflow characteristics. However, in the wind tunnel it was not possible to reproduce two characteristic phenomena observed in full-scale: dependence of integral length scales on reference wind velocity and a linear increase in integral length scales with height. Furthermore, in international standards there is a considerable scatter of recommended values for suburban wind characteristics. In particular, recommended integral length scales in ESDU 85020 (1985) are significantly larger than in other international standards. Truncated vortex generators applied in this study proved to be successful in part-depth suburban ABL wind-tunnel simulation that yield a novel methodology in studies on wind effects on structures and air pollution dispersion.
\end{abstract}

Keywords: atmospheric boundary layer; atmospheric turbulence; suburban country exposure; wind-tunnel experiments; experimental hardware; scaling issues; international standards and codes.

\section{Introduction}

There is a wide range of research tools for studies on natural wind characteristics, from field tests and wind-tunnel simulations to semi-empirical methods and numerical simulations using the Computational Fluid Dynamics (CFD). Field tests provide complete information on complex turbulent wind characteristics around buildings, whereas weather conditions cannot be fully controlled. Furthermore, wind environmental conditions can be measured only after the facility under scope has been constructed and no major changes in building design can be carried out. Wind-tunnel simulations enable controlled physical modelling of natural winds. However, they are not applicable for light wind conditions, issues related to scaling-down of atmospheric turbulence may arise, and they can be expensive and time consuming. Semi-empirical models are relatively

* Corresponding Author, Assistant Professor, E-mail: hkozmar@fsb.hr 
simple and easy-to-use with limited applicability and less accurate estimates. CFD simulations are less expensive than field- and wind-tunnel tests, more precise than semi-empirical models and they provide flow characteristics throughout the calculation domain simultaneously. However, CFD is less reliable in regard to maintaining turbulence characteristics along the numerical domain, although some significant improvements have been recently reported (Blocken et al. 2007, Hargreaves and Wright 2007, Yang et al. 2009). Advantages and drawbacks of different methods for natural wind simulation were recently reported in more detail by Blocken et al. (2008).

In wind-tunnel studies, one of major issues is the simulation length scale factor (e.g., Hunt 1982, Stathopoulos and Surry 1983). Smaller scale simulations, say 1:500, enable one to reproduce wind characteristics around entire high-rise structures and for larger areas. However, flow features cannot be reproduced in great detail. For larger simulation length scales, say 1:100, usually only the lower part of the ABL is modeled and that facilitates a better 'resolution' for wind characteristics around low-rise structures. For both types of ABL wind-tunnel simulation, it is very important to simultaneously reproduce the mean and turbulent wind structure, as turbulence is responsible for mixing and exchange of mass, heat and momentum throughout the ABL (Arya 2001). In general, simulation of turbulence is considered to be more essential than simulation of the mean wind profile (Tieleman 1990). Once natural winds have been reproduced in the wind tunnel, it is often compulsory to compare wind-tunnel results with recommended values in respective international codes and standards. However, an insight into these documents gives evidence about a considerable scatter of recommended values even though efforts have been made in order to harmonize international wind-loading standards and codes (e.g., Holmes et al. 2005).

In this study, scaling issues in wind-tunnel simulations of an atmospheric boundary layer developing above suburban areas were investigated. Two full-depth and one part-depth suburban ABL simulations were generated using the Counihan method: castellated barrier wall, vortex generators and surface roughness elements. Basic principles of full-depth and part-depth ABL windtunnel simulations are discussed in the next section. The truncated vortex generators, a modification of original Counihan design, were applied for a part-depth ABL simulation. An approach, applied in this study, has been previously tested for urban terrain exposures (Kozmar 2010). Experimental results were discussed in the light of wind specifications in respective international standards and codes.

\section{Experimental setup}

In general, the $\mathrm{ABL}$ can be reproduced in the wind tunnel as a full-depth or a part-depth simulation. Full-depth ABL simulations allow the entire depth of the ABL to be physically reproduced in the wind tunnel test section. They are commonly used in studies on wind loading of tall structures and dispersion of air pollutants. However, in full-depth simulations small-scale details of wind characteristics around low-rise structures cannot be precisely reproduced. If a low-rise structure is a topic of a wind-tunnel study, a part-depth simulation is commonly applied; it reproduces only the lower portion of the ABL, which facilitates a better 'resolution' for airflows around low-rise structures. Basic principles of full-depth and part-depth ABL wind-tunnel simulations are schematically shown in Fig. 1.

In this study, the suburban ABL flow was reproduced as two full-depth ABL simulations using the quarter-elliptic constant-wedge-angle vortex generators, a castellated barrier wall and a fetch of roughness elements originally developed by Counihan (1969a, 1969b, 1973), and one part-depth 


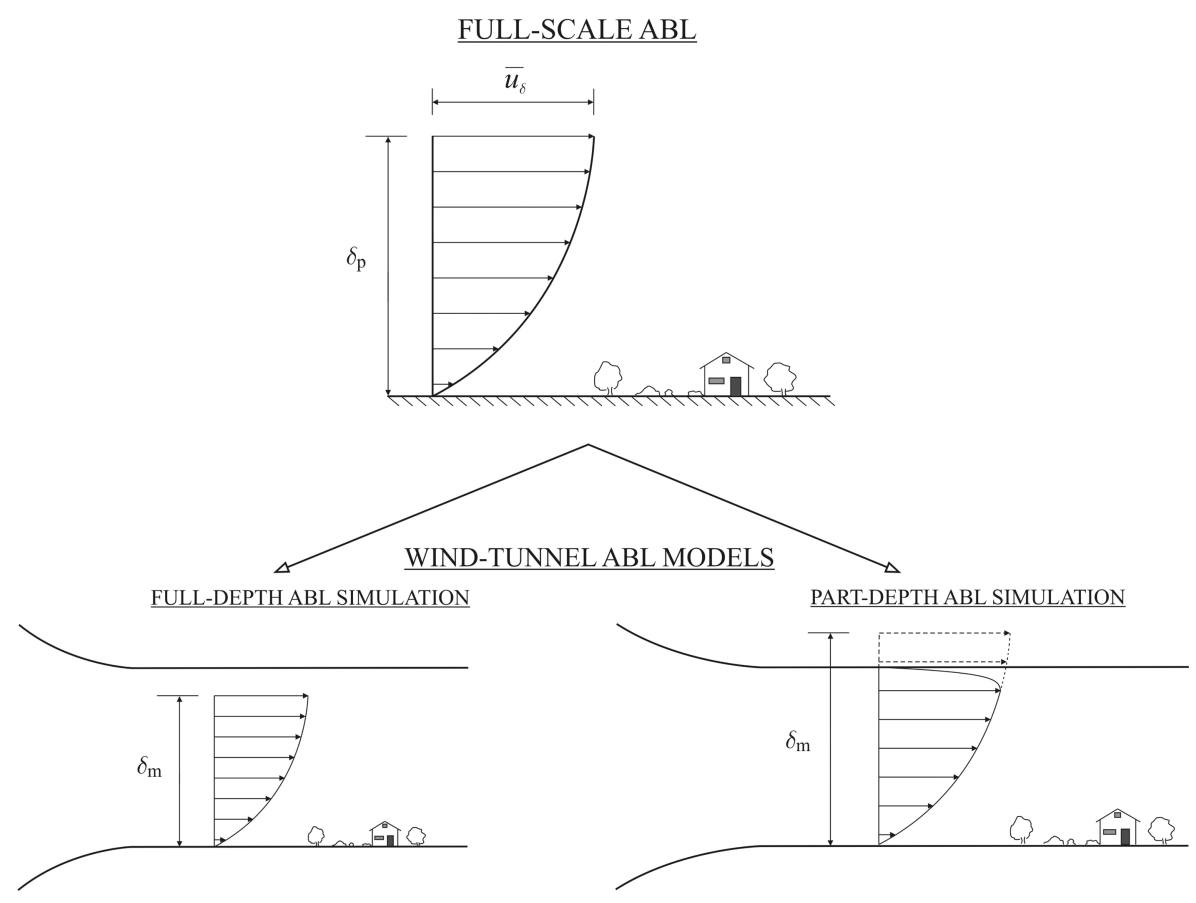

Fig. 1 Basic principles of full-depth and part-depth ABL wind-tunnel simulations

ABL simulation using the truncated vortex generators developed for the purposes of this study. Vortex generators in the 1:400, 1:250 and 1:220 simulations were $1 \mathrm{~m}, 1.5 \mathrm{~m}$ and $1.7 \mathrm{~m}$ high, respectively, as schematically reported in Kozmar (2010). In the 1:400 and 1:250 simulations vortex generators were manufactured using the original Counihan's design, and they were redesigned (truncated) for the 1:250 simulation. These truncated vortex generators already proved their applicability for part-depth ABL simulations in urban environments that was reported elsewhere (Kozmar 2010). Effects of ABL simulation devices on airflow characteristics in the wind tunnel, as well as characteristics of truncated vortex generators for different terrain types will be reported in due course. The simulation length scale factors, 1:400 and 1:250 in full-depth ABL simulations and 1:220 in the part-depth ABL simulation, were calculated as suggested by Cook (1978) using the aerodynamic roughness length $z_{0}$ and the integral length scale of turbulence ${ }^{x} L_{u}$.

LEGO cubes in a symmetrical, staggered pattern were used to simulate buildings and other obstructions in a suburban environment. The spacing and height of surface roughness elements in each configuration was preliminarily determined as indicated in Counihan (1971), Gartshore and De Croos (1977), and Fang and Sill (1992), and final arrangements were made by making continuous adjustments. Details of surface roughness and barriers' design in the 1:400, 1:250 and 1:220 suburban ABL wind-tunnel simulations are given in Table 1. Spacing density of surface roughness elements (test section area covered with surface roughness elements/total test section area) was $1.4 \%$ in all three configurations.

Effects of spacing between surface roughness elements on wind characteristics were previously reported in Kozmar (2008). Fetch was long enough to provide uniform flow conditions (Kozmar et al. 2005). Blockage in all tests was below 5\%, which allowed wind characteristics from the full- 
Table 1 Details of surface roughness and barriers' design in the 1:400, 1:250 and 1:220 suburban ABL windtunnel simulations

\begin{tabular}{cccc}
\hline \hline Simulation & $\begin{array}{c}\text { Height of surface } \\
\text { roughness elements }\end{array}$ & $\begin{array}{c}\text { Basic height of } \\
\text { barrier wall }\end{array}$ & $\begin{array}{c}\text { Height of barrier wall } \\
\text { castellation }\end{array}$ \\
\hline $1: 400$ & $20 \mathrm{~mm}$ & $127 \mathrm{~mm}$ & $42 \mathrm{~mm}$ \\
$1: 250$ & $70 \mathrm{~mm}$ & $220 \mathrm{~mm}$ & $63 \mathrm{~mm}$ \\
$1: 220$ & $70 \mathrm{~mm}$ & $292 \mathrm{~mm}$ & $83 \mathrm{~mm}$ \\
\hline
\end{tabular}

scale to be reproduced in the wind tunnel without deformation (e.g., Simiu and Scanlan 1996, Hucho 2002, Holmes 2007). Similarity criteria adopted in this study were previously applied in urban ABL wind-tunnel simulations (Kozmar 2010) and they were based on Plate (1982). Simulations were carried out assuming neutrally stable stratification of the ABL. In full-depth simulations (1:250 and 1:400) differences between recorded mean velocities $\bar{u}$ in top two measuring points were less than $1 \%$ indicating that $\mathrm{ABL}$ wind-tunnel models reached their gradient height at the top of vortex generators, which is in agreement with the boundary layer theory (Schlichting and Gersten 1997) and previous wind-tunnel tests (e.g., Farell and Iyengar 1999, Balendra et al. 2002, Kozmar 2010). Therefore, the boundary layer thickness $\delta_{\mathrm{m}}$ of $1 \mathrm{~m}$ and 1.5 $\mathrm{m}$ for 1:400 and 1:250 simulations was adopted, respectively. In the part-depth simulation $(1: 220)$ $\delta_{\mathrm{m}}$ would be $2 \mathrm{~m}$, as this would be the full height of non-truncated vortex generators. Scaling-up adopted values of $\delta_{\mathrm{m}}$, the full-scale ABL thickness $\delta_{\mathrm{p}}$ would be $400 \mathrm{~m}, 375 \mathrm{~m}$ and $440 \mathrm{~m}$ in the 1:400, 1:250 and 1:220 simulations, respectively, which agrees well with full-scale data reported for suburban type terrain (Counihan 1975). It needs to be mentioned that recent meteorological measurements reported ABL thickness of $1000 \mathrm{~m}$ and higher (e.g., Stull 2003), in which case the 1:400 and 1:250 simulations could be considered as part-depth ABL simulations as well. Nevertheless, in this study it was attempted to justify these two configurations as full-depth ABL simulations. Recently, valuable information on ABL characteristics was provided using Doppler sodar measuring technique (Tamura et al. 2007).

Experiments were carried out in a $1.80 \mathrm{~m}$ high, $2.70 \mathrm{~m}$ wide and $21 \mathrm{~m}$ long test section of the low-speed boundary layer wind tunnel ('Rudolf-Frimberger-Windkanal') at the Faculty of Mechanical Engineering, Technische Universität München (TUM). This Göttingen type wind tunnel is operable in a closed circuit and an open circuit mode with a suction configuration. In this study, experiments were conducted using the closed circuit mode. The adjustable ceiling enables a zero pressure gradient to be obtained along the wind tunnel test section. Structural models are usually placed at a turntable, whose centre is positioned $11.3 \mathrm{~m}$ downwind from the nozzle. The blower is driven by a $210 \mathrm{~kW}$ electric motor, which allows velocity regulation from $1 \mathrm{~m} / \mathrm{s}$ to $30 \mathrm{~m} / \mathrm{s}$. More technical details about this wind tunnel can be found elsewhere (e.g., Kozmar 2009). Characteristics of ABL wind-tunnel models were determined based on results of velocity measurements which were carried out using a triple hot-wire probe DANTEC 55P91 together with a ten-channel AALAB AN 1003 anemometer system. Velocity signals were sampled at $1.25 \mathrm{kHz}$ using a 12-bit digitizer Data Translation DT2821 for a total record length of $150 \mathrm{~s}$. Measurements were carried out in 21, 18 and 16 measuring points placed along a vertical line down the centre of the turntable at the scales of 1:400, 1:250 and 1:220, respectively. Tests were performed according to procedures proposed by Plate (1982) and Pernpeintner et al. (1995). 


\section{Characteristics of the suburban $A B L$ wind-tunnel simulations}

Values of characteristic parameters obtained in the 1:400, 1:250 and 1:220 suburban ABL simulations are reported in Table 2.

The displacement height $d$ and the aerodynamic surface roughness length $z_{0}$ were calculated by fitting measured mean velocities to the logarithmic-law and the power-law.

The power-law

$$
\frac{\bar{u}_{z}}{\bar{u}_{r e f}}=\left(\frac{z-d}{z_{r e f}-d}\right)^{\alpha}
$$

which is commonly used for representation of mean velocities in the $x$-direction.

The logarithmic-law

$$
\frac{\bar{u}_{z}}{u_{\tau}}=\frac{1}{\kappa} \ln \frac{z-d}{z_{0}}
$$

In this study, mean velocity in the $x$-direction $\bar{u}$ was calculated as

$$
\bar{u}=\frac{1}{T} \int_{0}^{T} u(t) \mathrm{d} t
$$

Turbulence intensity in the $x$-, $y$ - and $z$-direction

$$
I_{u}(z)=\frac{\sqrt{\overline{u^{\prime 2}(z)}}}{\bar{u}_{z}}, I_{v}(z)=\frac{\sqrt{\overline{v^{\prime 2}(z)}}}{\bar{u}_{z}}, I_{w}(z)=\frac{\sqrt{\overline{w^{\prime 2}(z)}}}{\overline{u_{z}}}
$$

respectively, where

$$
u(t)=\bar{u}+u^{\prime}(t), v(t)=\bar{v}+v^{\prime}(t) \text { and } w(t)=\bar{w}+w^{\prime}(t)
$$

Turbulent Reynolds shear stress

$$
\tau=-\rho \overline{u^{\prime} w^{\prime}}
$$

Integral length scales of turbulence

$$
{ }^{x} L_{u}=\int_{0}^{\infty} R_{u, x}(\Delta t) \mathrm{d} \Delta t,{ }^{x} L_{v}=\int_{0}^{\infty} R_{v, x}(\Delta t) \mathrm{d} \Delta t,{ }^{x} L_{w}=\int_{0}^{\infty} R_{w, x}(\Delta t) \mathrm{d} \Delta t
$$

Table 2 Values of characteristic parameters in the 1:400, 1:250 and 1:220 suburban ABL wind-tunnel simulations

\begin{tabular}{ccccccccc}
\hline \hline Simulation & $\delta_{\mathrm{m}}, \mathrm{m}$ & $\alpha_{\mathrm{m}}=\alpha_{\mathrm{p}}$ & $\mathrm{z}_{0 \mathrm{~m}}, \mathrm{~mm}$ & $\mathrm{z}_{0 \mathrm{p}}, \mathrm{m}$ & $d_{\mathrm{m}}, \mathrm{m}$ & $d_{\mathrm{p}}, \mathrm{m}$ & $u_{\text {rm }}, \mathrm{m} / \mathrm{s}$ & $u_{\text {tp }}, \mathrm{m} / \mathrm{s}$ \\
\hline $1: 400$ & 1.0 & 0.21 & 0.45 & 0.18 & 0.01 & 4.0 & 0.63 & 0.96 \\
$1: 250$ & 1.5 & 0.21 & 0.96 & 0.24 & 0.03 & 7.5 & 1.06 & 1.57 \\
$1: 220$ & $2.0^{*}$ & 0.21 & 1.59 & 0.35 & 0.02 & 4.5 & 1.17 & 1.71 \\
\hline
\end{tabular}

*Originally, the full boundary layer thickness in the 1:220 simulation would be $2.0 \mathrm{~m}$. However, in this configuration only the lower portion of the boundary layer was simulated 
calculated using autocorrelation functions and assuming the validity of Taylor's hypothesis (frozen turbulence); details of this procedure can be found in Simiu and Scanlan (1996).

Power spectral density of longitudinal velocity fluctuations $S_{u}(f)$, where

$$
\overline{u^{\prime 2}}=\int_{0}^{\infty} S_{u}(f) \mathrm{d} f
$$

Fig. 2 shows recorded mean velocities $\bar{u}_{z}$ compared to fitted power-law curve with the power-law exponent $\alpha=0.21$. Mean velocities $\bar{u}_{z}$ at height $z$ were normalized with the mean reference velocity $\bar{u}_{\text {ref }}$ at height $z_{\text {ref }}$. In the 1:400, 1:250 and 1:220 simulations $z_{\text {ref }}$ was $48 \mathrm{~m}, 54 \mathrm{~m}$ and $53 \mathrm{~m}$ fullscale, respectively. Originally, it was attempted to use $50 \mathrm{~m}$ full-scale as a reference height. However, as these reference heights were scaled-up from the wind tunnel using the wind-tunnel measuring points and adopted length scale factors, it was not possible to obtain exactly the same reference height in all simulations. Nevertheless, it seems reasonable to accept reference heights 48 $\mathrm{m}, 54 \mathrm{~m}$ and $53 \mathrm{~m}$ full-scale for the purposes of this study, as they do not differ significantly from aimed $50 \mathrm{~m}$ height full-scale.

In general, experimental results agree very well with the power-law for $\alpha=0.21$, which can be accepted for representation of an atmospheric boundary layer developing above suburban type terrain. In ESDU 72026 (1972) power-law exponents between $\alpha=0.20$ and $\alpha=0.23$ were reported for aerodynamic roughness lengths $z_{0 \mathrm{p}}$ between $0.1 \mathrm{~m}$ and $0.3 \mathrm{~m}$, respectively, representing terrain with more numerous small obstructions such as hedges, trees and buildings (e.g., typical farmland). In two full-depth ABL simulations (1:400 and 1:250) recorded mean velocities above $200 \mathrm{~m}$ slightly exceed the power-law values. In the part-depth ABL simulation (1:220), results were reported only up to $300 \mathrm{~m}$, as only the lower portion of the ABL was reproduced in the wind tunnel. Values of $z_{0 \mathrm{p}}$ and $d_{\mathrm{p}}$ reported in Table 2 were scaled-up from $z_{0 \mathrm{~m}}$ and $d_{\mathrm{m}}$ using the length scale factors 1:400, 1:250 and 1:220. In this study, $z_{0 \mathrm{p}}$ and $d_{\mathrm{p}}$ agree well with typical full-scale values. For outskirts of small towns, villages, countryside with many hedges, some trees and some

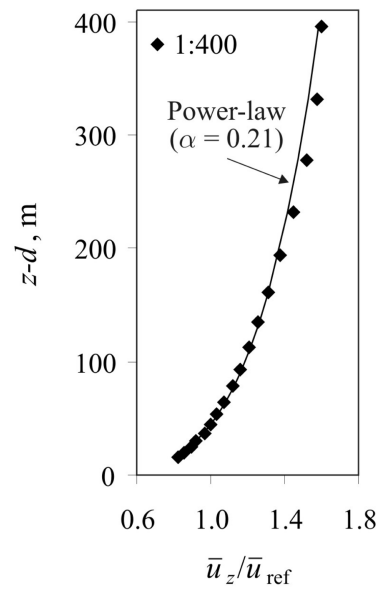

(a)

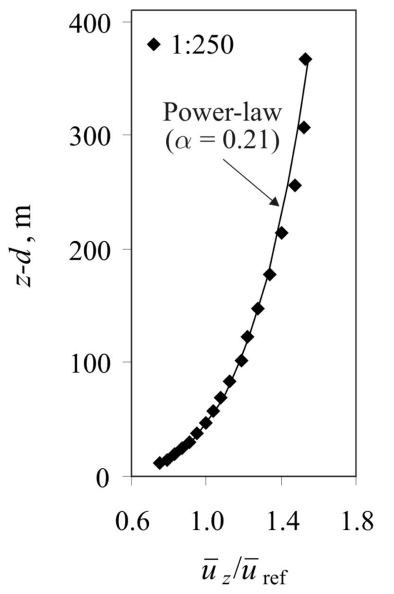

(b)

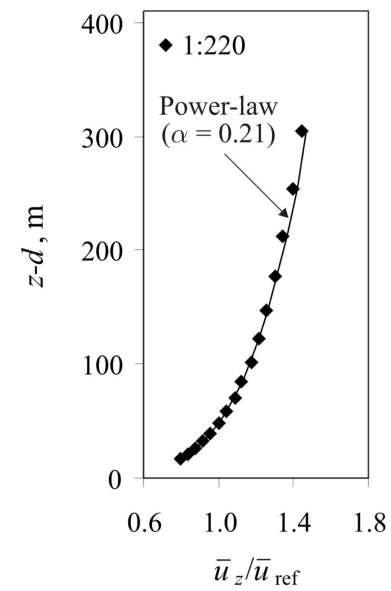

(c)

Fig. 2 Mean velocity profiles in the 1:400, 1:250 and 1:220 suburban ABL wind-tunnel simulations; linear presentation 
buildings ESDU 85020 (1985) reports values around $z_{0 \mathrm{p}}=0.1 \mathrm{~m}$ and $d=0 \mathrm{~m}$ to $2 \mathrm{~m}$. For small towns, suburbs of large towns and cities, wooded country with many trees ESDU 85020 (1985) suggests $z_{0 \mathrm{p}}=0.3 \mathrm{~m}$ and $d=5 \mathrm{~m}$ to $10 \mathrm{~m}$. Holmes (2007) indicated that for a suburban terrain with buildings' height between $3 \mathrm{~m}$ and $5 \mathrm{~m}$ aerodynamic roughness length could be between $0.1 \mathrm{~m}$ and $0.5 \mathrm{~m}$. Simiu and Scanlan (1996) report $z_{0 \mathrm{p}}$ for sparsely built-up suburbs could be between $0.2 \mathrm{~m}$ and $0.4 \mathrm{~m}$. The friction velocities $u_{\mathrm{mm}}$ calculated indirectly from the logarithmic mean velocity profile were $0.63 \mathrm{~m} / \mathrm{s}, 1.06 \mathrm{~m} / \mathrm{s}$ and $1.17 \mathrm{~m} / \mathrm{s}$ in the 1:400, 1:250 and 1:220 simulations, respectively. When scaling-up these values using the procedure suggested in Simiu and Scanlan (1996) originally developed as a relation between wind speeds in different roughness regimes

$$
\frac{u_{\tau \mathrm{m}}}{u_{\tau \mathrm{p}}}=\left(\frac{z_{0 \mathrm{~m}}}{z_{0 \mathrm{p}}}\right)^{0.0706}
$$

the friction velocities $u_{\tau \mathrm{p}}$ are $0.96 \mathrm{~m} / \mathrm{s}, 1.57 \mathrm{~m} / \mathrm{s}$ and $1.71 \mathrm{~m} / \mathrm{s}$ for the $1: 400,1: 250$ and $1: 220$ simulations, respectively, indicating slightly different friction intensity between the surface and the air in different configurations. The 1:220 simulation has the roughest surface, as both $u_{\tau \mathrm{p}}$ and $z_{0 \mathrm{p}}$ full-scale values were maximal in this configuration indicating that simulated near-ground atmospheric turbulence in this configuration could be stronger than in two other simulations. Furthermore, the scaled-up friction velocities $\left(u_{\tau \mathrm{p}}\right)$ coincide well with typical full-scale values reported in Dyrbye and Hansen (1997), which are between $1 \mathrm{~m} / \mathrm{s}$ and $2 \mathrm{~m} / \mathrm{s}$. It should be noted that the approach suggested in Eq. (9) is valid only for uniform roughness and sufficiently large fetch.

A comparison of experimental results with the logarithmic-law is reported in Fig. 3. Mean velocities $\bar{u}_{z}$ normalized with corresponding friction velocities $u_{\tau \mathrm{m}}$ reported in Table 2 agree well with the logarithmic-law throughout the inertial sublayer and even beyond it, up to approximately $150 \mathrm{~m}$. Moreover, Sockel (1984) indicated that the logarithmic-law can be successfully applied only up to $150 \mathrm{~m}$ height and for longer time records. Paralleling comparison of experimental results with the power-law presented in Fig. 2, recorded mean velocities in two full-depth ABL simulations (1:400 and 1:250) above $200 \mathrm{~m}$ slightly exceed the logarithmic-law values. In general, experimental results reported in Figs. 2 and 3 clearly indicate it is possible to generate nearly the same mean airflows for different simulation length scale factors.

Characteristics of simulated suburban atmospheric turbulence were studied based on wind-tunnel results for turbulence intensity, turbulent Reynolds shear stress, integral length scale of turbulence, and power spectral density of velocity fluctuations. Fig. 4 shows turbulence intensity profiles $I_{u}, I_{v}$ and $I_{w}$ in the 1:400, 1:250 and 1:220 suburban ABL wind-tunnel simulations.

Turbulence intensities $I_{u}, I_{v}$ and $I_{w}$, reported in Fig. 4 for each configuration separately, show good agreement with the equation

$$
I_{u}: I_{v}: I_{w}=1: 0.75: 0.5
$$

suggested by Counihan (1975). Experimental results agree well with equation proposed by Holmes (2007) as well, who related turbulence intensities in the $x$-, $y$ - and $z$-direction using the form

$$
I_{u}: I_{v}: I_{w}=1: 0.88: 0.55
$$

based on full-scale measurements. These full-scale measurements (Holmes 2007) also proved that 

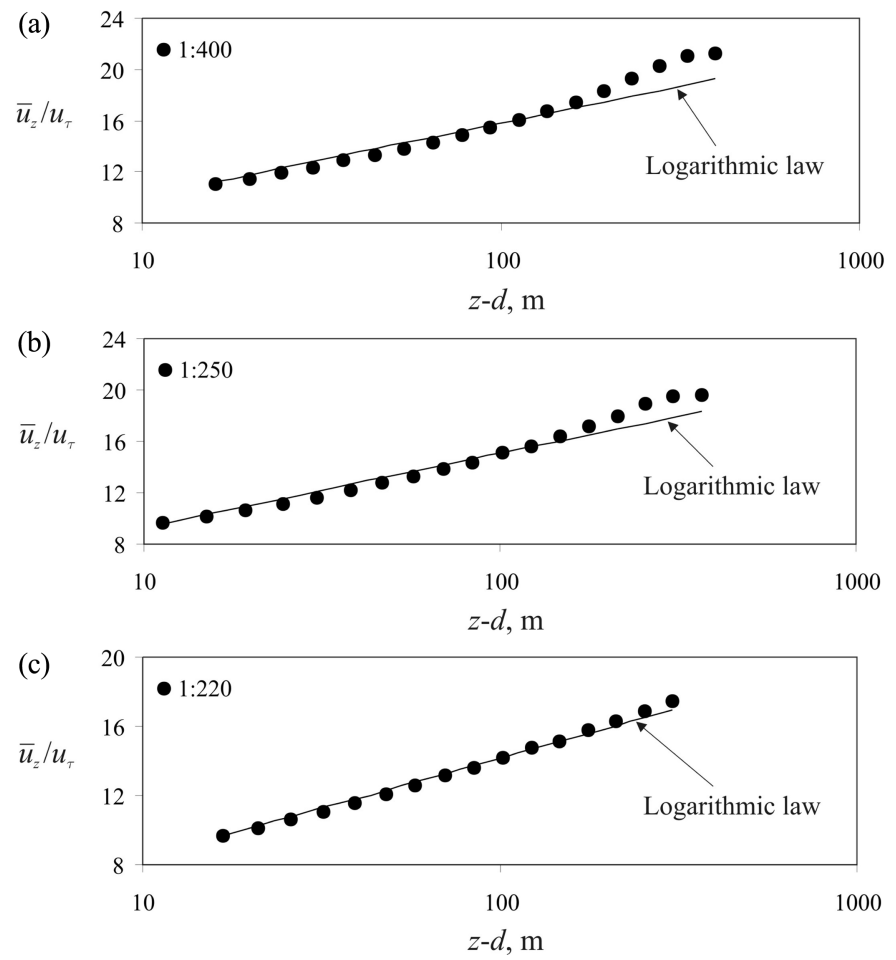

Fig. 3 Mean velocity profiles in the 1:400, 1:250 and 1:220 suburban ABL wind-tunnel simulations, logarithmic presentation

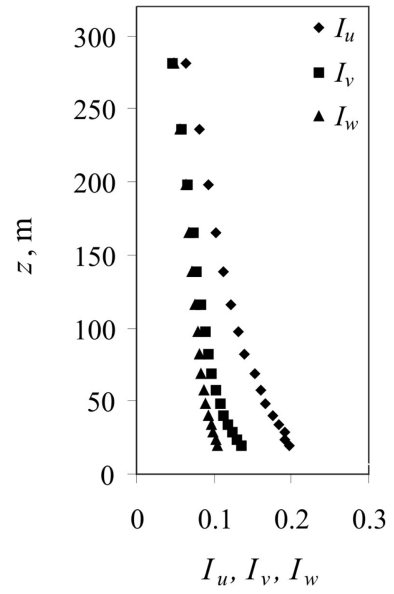

(a)

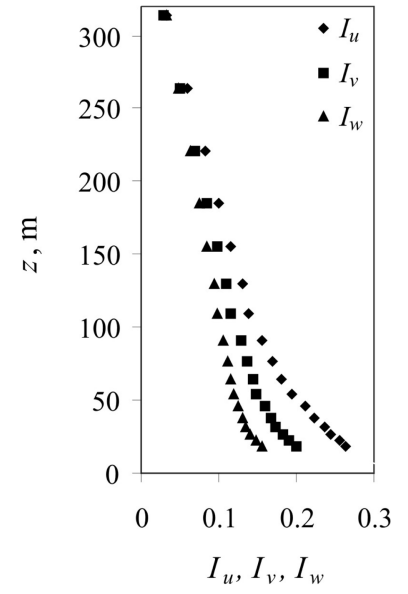

(b)

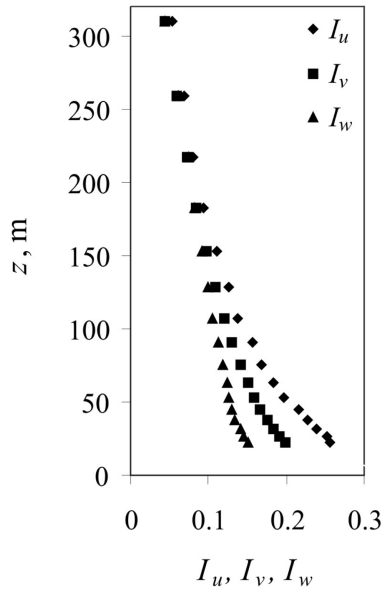

(c)

Fig. 4 Turbulence intensity profiles $I_{u}, I_{v}$ and $I_{w}$ in the (a) 1:400, (b) 1:250 and (c) 1:220 suburban ABL windtunnel simulations

the standard deviation of longitudinal wind speed $\left(\sigma_{u}\right)$ is equal to $2.5 u_{\tau}$. Furthermore, Holmes (2007) suggested a relationship 


$$
I_{u}=\frac{1}{\ln \left(\frac{z}{z_{0}}\right)}
$$

which is implemented in ISO 4354 (1997) and Eurocode EN 1991-1-4 (2005) for calculation of full-scale turbulence intensity $I_{u}$ as well. Figs. 5, 6 and 7 show turbulence intensity profiles $I_{u}, I_{v}$ and $I_{w}$, respectively. Wind-tunnel results were scaled-up using the scale factors 1:400, 1:250 and 1:220, and compared to ESDU 74031 (1974) data. The ESDU 74031 (1974) boundaries are represented as $\pm 20 \%$ from ESDU 74031 (1974) recommended values for aerodynamic roughness length $z_{0}=0.1 \mathrm{~m}$

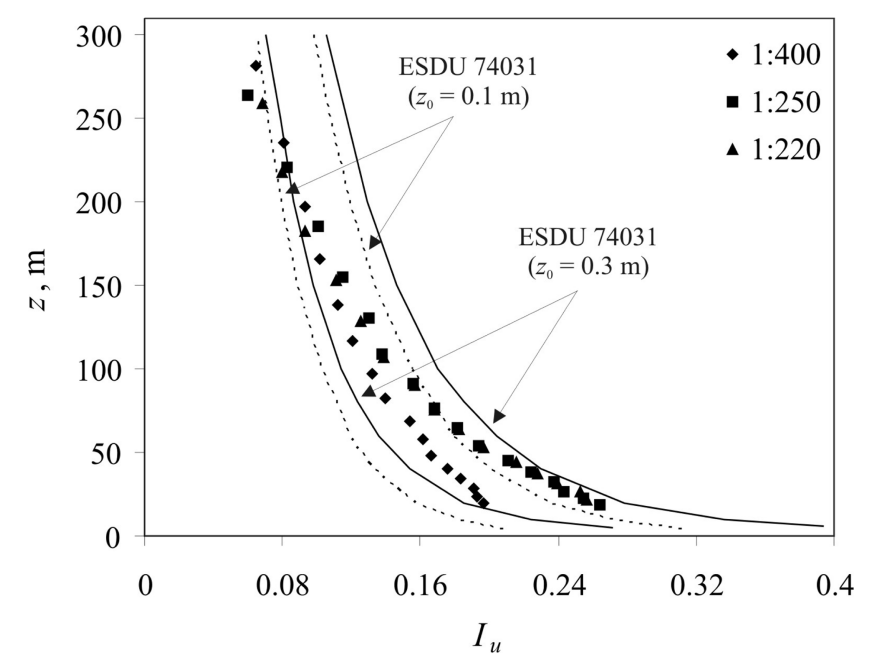

Fig. 5 Profiles of the longitudinal turbulence intensity in the 1:400, 1:250 and 1:220 suburban ABL windtunnel simulations

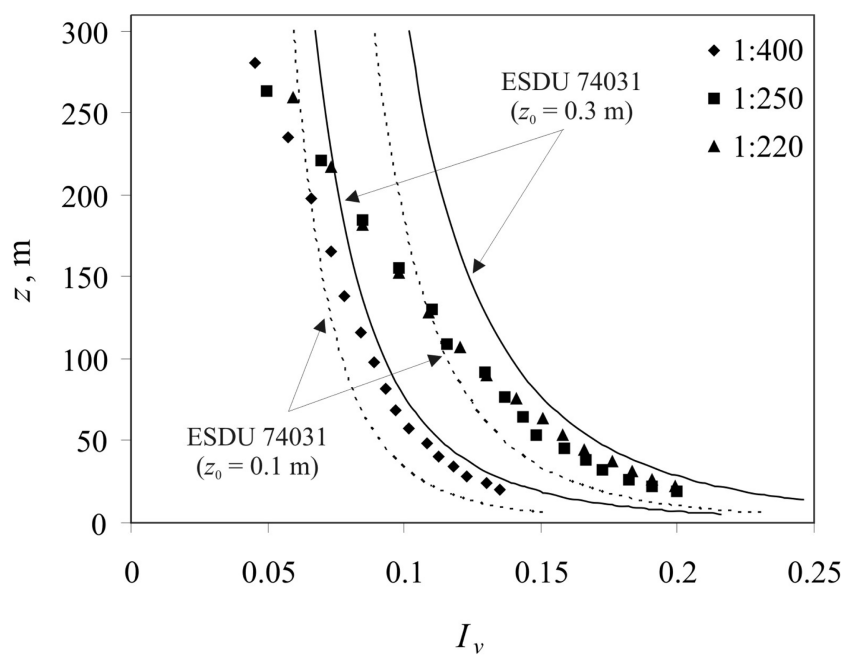

Fig. 6 Profiles of the lateral turbulence intensity in the 1:400, 1:250 and 1:220 suburban ABL wind-tunnel simulations 


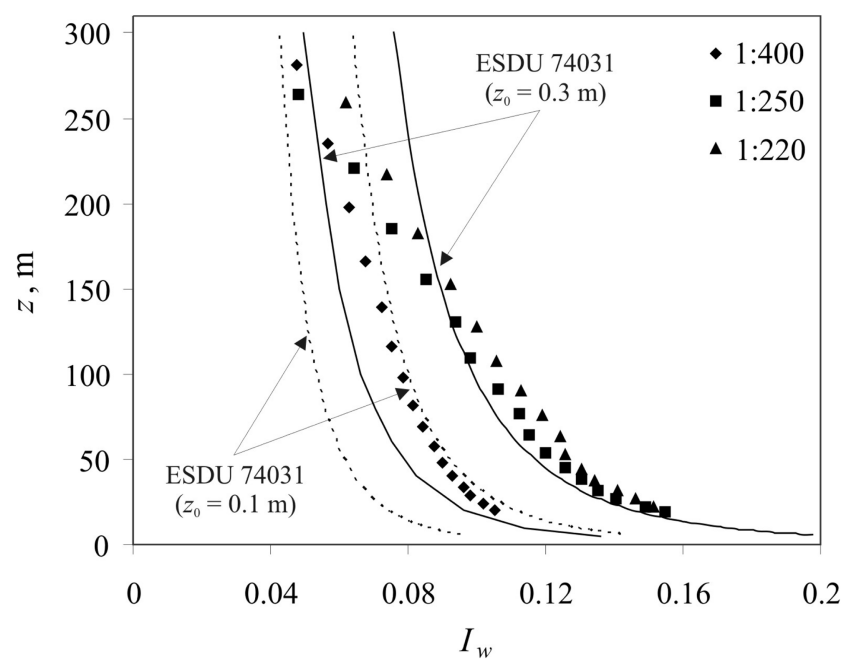

Fig. 7 Profiles of the vertical turbulence intensity in the 1:400, 1:250 and 1:220 suburban ABL wind-tunnel simulations

full-scale and $z_{0}=0.3 \mathrm{~m}$ full-scale; ESDU 85020 (1985) indicates a tolerance range of $\pm 20 \%$ can be accepted for practical purposes. Due to difficulties concerning velocity measurements in the upper ABL, ESDU 74031 (1974) data are available only up to $300 \mathrm{~m}$. In this section, obtained turbulence parameters were compared to the 'old' ESDU 74031 (1974), where the wind specifications are not dependent on mean wind speeds, i.e., there is only one value of turbulence intensity and integral length scale of turbulence at each height for a specific terrain exposure. Furthermore, the ESDU 74031 (1974) is compared with the 'new' ESDU 85020 (1985) in the next section, as well as with other major international standards.

In general, a good agreement between the wind-tunnel results and ESDU 74031 (1974) data was obtained. Turbulence intensities $I_{u}, I_{v}$ and $I_{w}$ in the 1:400 simulation are lower compared to the 1:250 and 1:220 simulations due to the least rough terrain in this configuration, as reported in Table 2. Effects of surface roughness on turbulence intensity profiles are stronger close to surface. Vertical turbulence intensities $I_{w}$ are larger than ESDU 74031 (1974) data for $z_{0}=0.3 \mathrm{~m}$ closer to surface in the 1:250 and 1:220 simulations, in agreement with a trend reported in Wang et al. (1996). Generally, vertical turbulence intensities in all three ABL simulations tend to be slightly larger than suggested in Eqs. (10) and (11).

Although knowledge of the turbulent Reynolds shear stress in applications to wind loading on ground-based structures is only required in special cases (ESDU 85020 1985), it seems useful to compare experimental results with ESDU 85020 (1985) to further validate an approach presented in this study. Previously, Cermak et al. (1995) demonstrated that vertical distribution of Reynolds shear stress may be successfully modelled in the wind tunnel. In general, Reynolds stress represents a measure for upwind transport of retarding forces exerted on the wind at the Earth's surface. The Reynolds stresses $-\rho \overline{u^{\prime} v^{\prime}}$ and $-\rho \overline{v^{\prime} w^{\prime}}$ tend to be very small, and can be ignored in practice (ESDU 85020 1985). Experimental wind-tunnel results for turbulent Reynolds shear stress $-\rho \overline{u^{\prime} w^{\prime}}$ are reported in Fig. 8 and compared to ESDU 74031 (1974) data up to $300 \mathrm{~m}$ full-scale for $z_{0}=0.1 \mathrm{~m}$, $0.3 \mathrm{~m}$ and $0.5 \mathrm{~m}$ full-scale.

It can be observed that experimental results agree fairly well between the 1:400, 1:250 and 1:220 


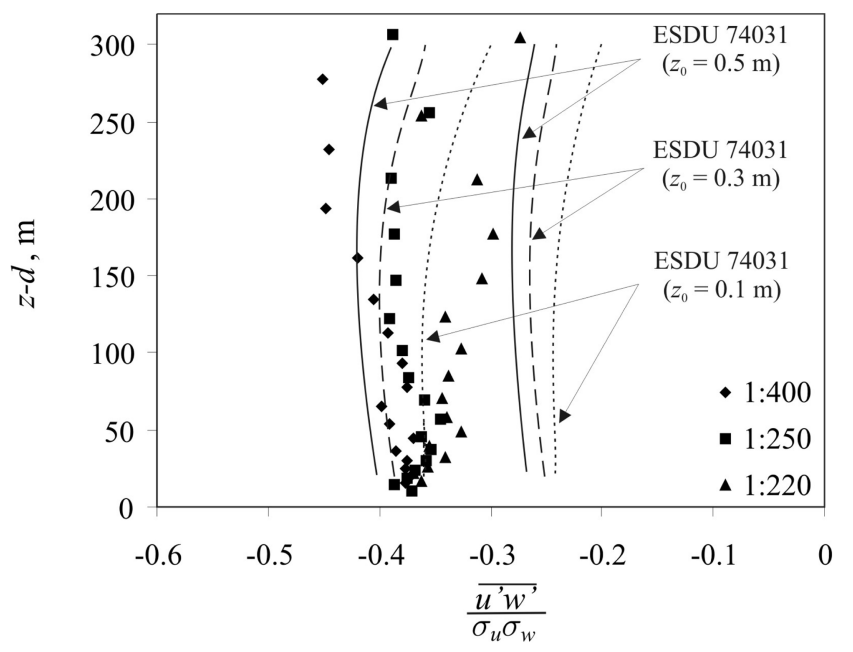

Fig. 8 Turbulent Reynolds shear stress profiles in the 1:400, 1:250 and 1:220 suburban ABL wind-tunnel simulations

simulations in the near-ground region. Higher than $100 \mathrm{~m}$ Reynolds stresses recorded in different configurations show larger discrepancies. However, for a wind engineer a primarily focus of interest is lower $100 \mathrm{~m}$ to $200 \mathrm{~m}$ within an ABL, as this is the height range of most structures (Holmes 2007). In general, experimental values reported in Fig. 8 agree well with the ESDU 74031 (1974) data given for $z_{0}=0.5 \mathrm{~m}$ full-scale, which is slightly higher than aerodynamic length scales obtained in this study $\left(0.18 \mathrm{~m}<z_{0}<0.35 \mathrm{~m}\right)$. However, in ESDU 85020 (1985) it was indicated that Reynolds stresses reported in the literature may be slightly higher than the ESDU values due to response characteristics of typical anemometers.

Figs. 9, 10 and 11 show profiles of the ${ }^{x} L_{u},{ }^{x} L_{v}$ and ${ }^{x} L_{w}$ integral length scales of turbulence, respectively. Experimental results were calculated as suggested in Eq. (7) and they were compared to the ESDU 74031 (1974) data assuming there were no roughness changes upwind of the measuring site. In general, integral length scales in the wind tunnel calculated using autocorrelations functions tend to be slightly larger than the length scales calculated using the peak of the power spectra (Flay and Stevenson 1988, Kozmar 2010). The area between the lines represents a range within $\pm 30 \%$ tolerance band reported in the ESDU 74031 (1974) for aerodynamic roughness length $z_{0}=0.1 \mathrm{~m}$ full-scale and $z_{0}=0.3 \mathrm{~m}$ full-scale. Wind-tunnel results were scaled-up using the adopted simulation length scale factors 1:400, 1:250 and 1:220. Due to difficulties with full-scale velocity measurements in the upper ABL, the ESDU 74031 (1974) integral length scales were available only up to $300 \mathrm{~m}$ full-scale.

Integral length scale profiles for the 1:400, 1:250 and 1:220 suburban ABL wind-tunnel simulations agree fairly well to each other, especially in the lower $100 \mathrm{~m}$ full-scale, indicating a possibility to reproduce similar turbulent structures at different simulation length scales. Similar tendency was observed for the ${ }^{x} L_{u}$ integral length scales of turbulence in urban ABL wind-tunnel simulations (Kozmar 2010). Reported experimental results agree moderately with the ESDU 74031 (1974) data. However, a linear increase in integral length scales of turbulence with height, which would be similar to full-scale (Arya 1999), was not observed. In this study, experimental results strongly increase in the near-ground region but with further increase in height they remain nearly 
constant. This trend was reported in other wind-tunnel studies as well (e.g., Farell and Iyengar 1999) and is possibly due to different mechanisms of boundary layer development in the wind tunnel and in the full-scale (Kozmar 2010). Furthermore, it is particularly difficult to incorporate larger eddies into the wind-tunnel boundary layer (Peterka et al. 1998, Lim et al. 2007), as the confined cross-section dimensions of the wind tunnel test section do not allow large eddies fully to develop. Moreover, largest eddies in the wind tunnel have roughly the size of the same order as the test section dimensions. The ${ }^{x} L_{u},{ }^{x} L_{v}$ and ${ }^{x} L_{w}$ integral length scales of turbulence in the 1:220 simulation are smaller than in two other simulations, especially in the upper part of the suburban

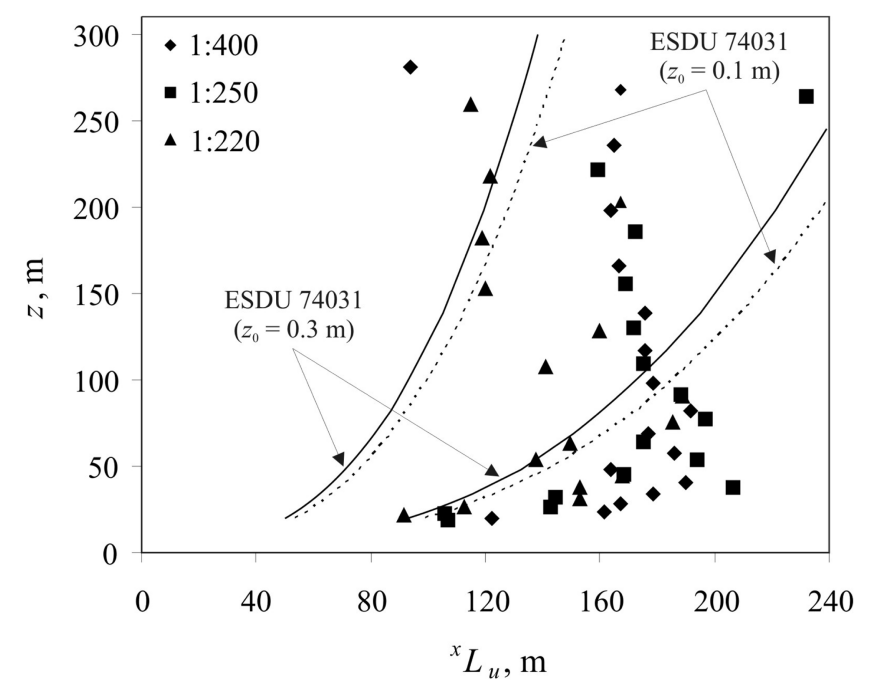

Fig. 9 Integral length scales of turbulence ${ }^{x} L_{u}$ for the 1:400, 1:250 and 1:220 suburban ABL wind-tunnel simulations

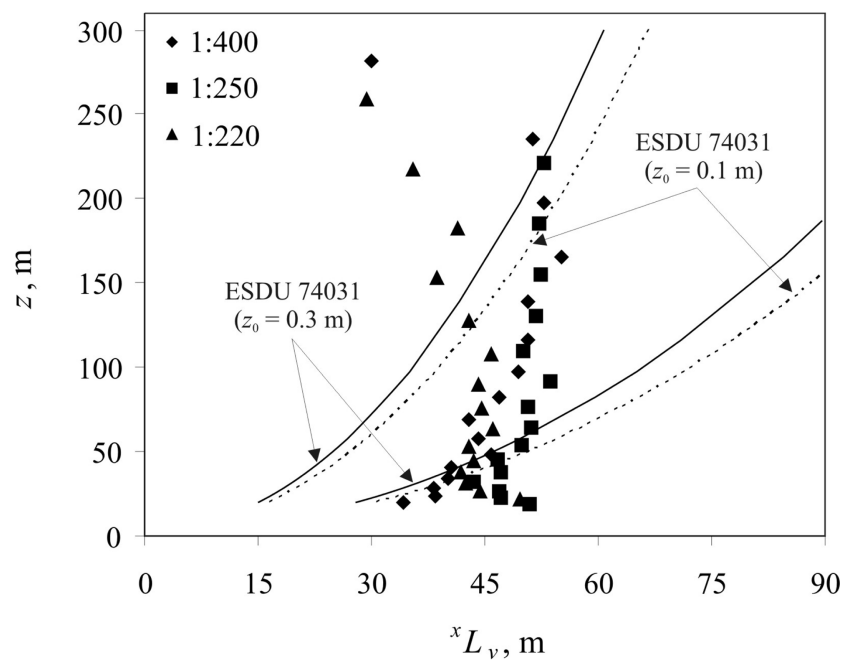

Fig. 10 Integral length scales of turbulence ${ }^{x} L_{v}$ for the 1:400, 1:250 and 1:220 suburban ABL wind-tunnel simulations 


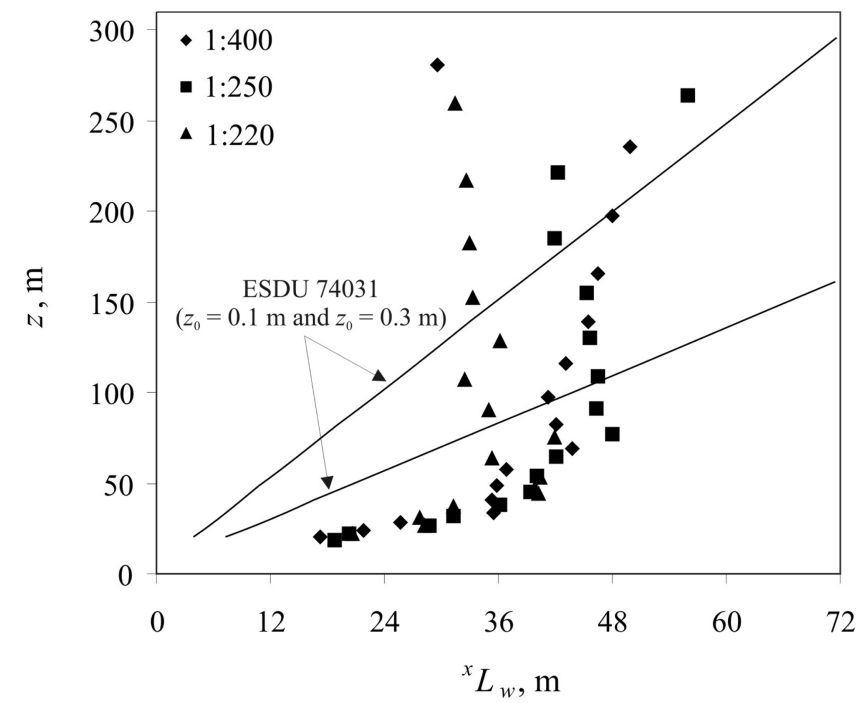

Fig. 11 Integral length scales of turbulence ${ }^{x} L_{w}$ for the 1:400, 1:250 and 1:220 suburban ABL wind-tunnel simulations

ABL model, possibly due to rougher terrain in this simulation $\left(z_{0}=0.35 \mathrm{~m}\right.$ full-scale). Furthermore, in ESDU 74031 (1974) it was reported that 'length scales of turbulence are influenced by surface roughness, being smaller for rough terrain (small eddies of high intensity) and larger for smooth terrain (larger eddies of relatively low intensity)'.

Figs. 12, 13 and 14 show power spectra of longitudinal velocity fluctuations measured in the 1:400, 1:250 and 1:220 simulations, which were calculated using the fast Fourier transformations as suggested by Bendat and Piersol (2000).

Results are reported for two different full-scale heights in each simulation; $24 \mathrm{~m}$ and $82 \mathrm{~m}$ in the 1:400 simulation, $22.5 \mathrm{~m}$ and $77 \mathrm{~m}$ in the $1: 250$ simulation, $26.5 \mathrm{~m}$ and $90 \mathrm{~m}$ in the $1: 220$ simulation. Measurement points were selected in a way to give insight into turbulence characteristics in near-ground region (heights around $25 \mathrm{~m}$ ), and higher up in the ABL models

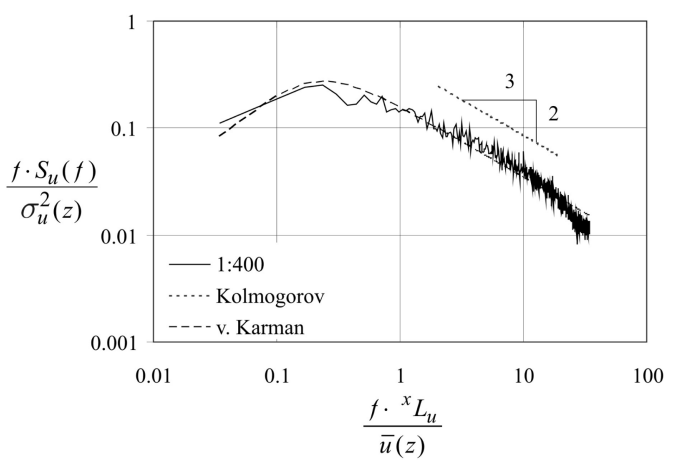

(a)

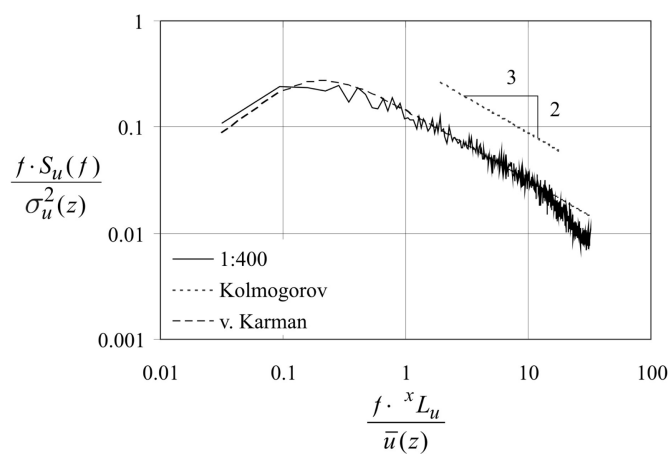

(b)

Fig. 12 Power spectral density of longitudinal velocity fluctuations: (a) $z=24 \mathrm{~m}$ full-scale and (b) $z=82 \mathrm{~m}$ full-scale in the 1:400 suburban ABL simulation 


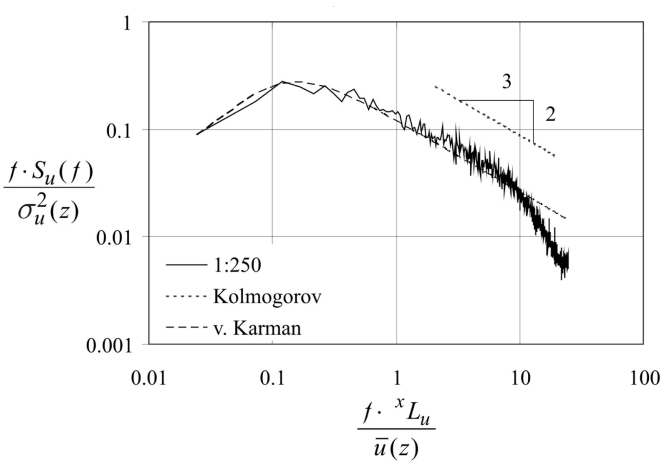

(a)

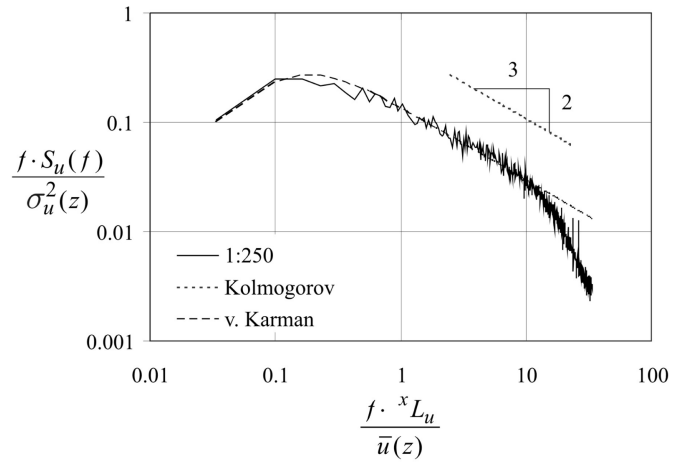

(b)

Fig. 13 Power spectral density of longitudinal velocity fluctuations: (a) $z=22.5 \mathrm{~m}$ full-scale and (b) $z=77 \mathrm{~m}$ full-scale in the 1:250 suburban ABL simulation

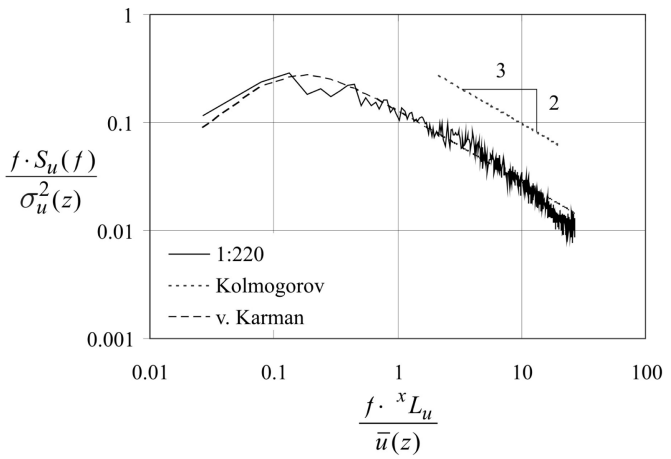

(a)

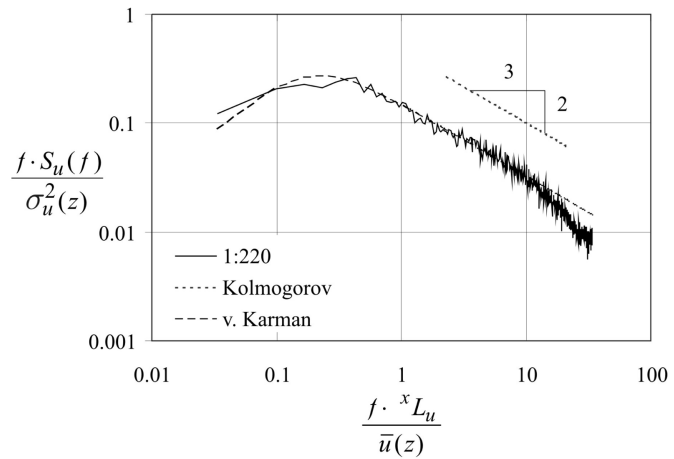

(b)

Fig. 14 Power spectral density of longitudinal velocity fluctuations: (a) $z=26.5 \mathrm{~m}$ full-scale and (b) $z=90 \mathrm{~m}$ full-scale in the 1:220 suburban ABL simulation

(heights between $70 \mathrm{~m}$ and $100 \mathrm{~m}$ ). For low-rise buildings it is particularly important to correctly reproduce atmospheric turbulence in the near-ground region to enable improving wind environment around buildings in regard to wind loading and air quality. Furthermore, Tieleman (2003) indicated that both the high- and the low-frequency segments of the velocity spectra need to be simulated in order to adequately reproduce the surface pressures under separated shear layers on bluff buildings. The turbulence integral length scale represents the large-scale turbulence, while the small-scale turbulence is represented with the small-scale turbulence parameter originally introduced by Melbourne (1979), defined (for the present case) by $S=f \cdot S_{u}(f) / U_{h}^{2}$ evaluated at $f=10 U_{h} / h$; $S_{u}(f)$ is the longitudinal spectral energy density at frequency $f$ and $z=h$, and $U_{h}$ is the mean velocity at $z=h$. In this study, Melbourne's small-scale turbulence parameter is within a factor 2 at lower heights (Figs. 12(a), 13(a), 14(a)) and within a factor 5 for results reported in Figs. 12(b), 13(b), 14(b). Lim et al. (2007) reported a similar mismatch in small-scale turbulence parameter indicating that it is not possible to match both the large-scale and the small-scale turbulence simultaneously due to, in part, Reynolds number effects. Power spectral density profiles in all three wind-tunnel simulations agree well with the theoretical models of Kolmogorov (1941) and von 
Kármán (1948), which are commonly used to represent atmospheric turbulence. The obtained power spectra reach maximum at reduced frequencies between 0.1 and 0.3 and they follow the Kolmogorov $2 / 3$ slope in the inertial subrange indicating a similar path in turbulent boundary layer structure not dependent on the simulation length scale. In the 1:250 simulation power spectra die-off quicker at higher frequencies than in two other simulations, that is to be investigated in more detail in the future.

\section{Characteristics of the suburban $A B L$ in international standards}

In wind-tunnel studies it is often compulsory to compare recorded wind-tunnel results with recommended values in international wind loading codes and standards. However, there is a considerable scatter of values reported in these documents. Therefore, results from this study were compared to several major international standards to shed more light on this issue. Profiles of the longitudinal turbulence intensity $I_{u}$ and integral length scales of turbulence ${ }^{x} L_{u}$ are shown in Figs. 15 and 16, respectively, together with values recommended in ESDU 74031 (1974), ESDU 85020 (1985), AIJ (1996), ISO 4354 (1997), Eurocode EN 1991-1-4 (2005) and ASCE 7-05 (2006). It should be noted that turbulence intensities in ISO 4354 (1997) are defined as in Eurocode EN 19911-4 (2005). Furthermore, in ISO 4354 (1997) recommended values for integral length scales of turbulence are not available. Values for both ESDUs, i.e., 74031 and 85020, were reported in order to highlight their differences. In Figs. 15 and 16, the spacing between the lines represents $\pm 20 \%$ tolerance band for turbulence intensities and $\pm 30 \%$ for integral length scales of turbulence, as calculated from the recommended values in respective standards.

Turbulence intensities $I_{u}$ in ESDU 74031 (1974), ESDU 85020 (1985), ISO 4354 (1997) and Eurocode EN 1991-1-4 (2005) were calculated for $z_{0}=0.3 \mathrm{~m}$, in AIJ (1996) for exposure III. Integral length scales of turbulence ${ }^{x} L_{u}$ were calculated for $z_{0}=0.3 \mathrm{~m}$ in all standards, except in AIJ (1996) where the turbulence scale is defined independently of the terrain type. It needs to be mentioned that exposures B and C in ASCE 7-05 (2006) do not exactly match suburban ABL

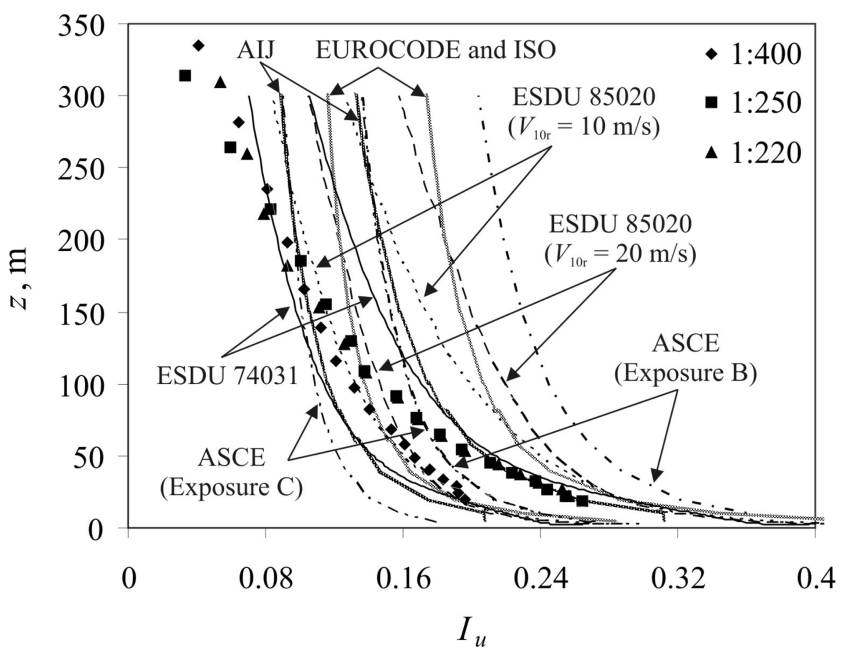

Fig. 15 Profiles of the longitudinal turbulence intensity $I_{u}$ in the 1:400, 1:250 and 1:220 suburban ABL windtunnel simulations compared to values recommended in international standards 
models in this study. Mean hourly wind-speed power-law exponent $\bar{\alpha}$ was 0.25 for exposure B and 0.15 for exposure C in ASCE 7-05 (2006), while in this study $\alpha$ was 0.21 in all simulations. Values in all reported standards, except ESDU 85020 (1985), do not depend on mean wind velocity. Harris (1986) indicated that the size of turbulent eddies close to the ground varies primarily with changes in surface roughness, and that their dependence on reference velocity recommended in ESDU 85020 (1985) is a result of similarity principles, including an increase in the ABL thickness with wind velocity. Furthermore, Harris (1986) implies that integral length scales of turbulence at design wind velocities are larger than at the wind velocities at which measurements are typically taken.

Experimental results for turbulence intensity $I_{u}$ shown in Fig. 15 agree up to $100 \mathrm{~m}$ with ESDU 85020 (1985) for $V_{10 \mathrm{r}}=10 \mathrm{~m} / \mathrm{s}$, ISO 4354 (1997), Eurocode EN 1991-1-4 (2005), and even beyond it with ESDU 74031 (1974) and AIJ (1996). In this region experimental results are between ASCE 7-05 (2006) bandwidths for exposure B and C, which was expected due to a fact that exposure B is more-urban and exposure $\mathrm{C}$ is more-rural than the simulations generated in this study. Recommended values in ESDU 85020 (1985) for reference wind velocity $V_{10 \mathrm{r}}=20 \mathrm{~m} / \mathrm{s}$ are larger than the values in ESDU $85020(1985)$ for $V_{10 \mathrm{r}}=10 \mathrm{~m} / \mathrm{s}$ and in other international standards for suburban type terrain characterized with the power-law exponent $\alpha=0.21$. Therefore, recommended values in ESDU 85020 (1985) are larger than in the preceded ESDU 74031 (1974). However, ESDU 85020 (1985) values approach those from ESDU 74031 (1974) for smaller reference wind velocities.

Integral length scales of turbulence ${ }^{x} L_{u}$ reported in Fig. 16 agree up to $100 \mathrm{~m}$ with ASCE 7-05 (2006) for both exposures B and C. However, in near-ground region results are slightly larger than ESDU 74031 (1974), AIJ (1996), Eurocode EN 1991-1-4 (2005). Except in lowest $50 \mathrm{~m}$, where obtained ${ }^{x} L_{u}$ values fall within ESDU 85020 (1985) boundaries for both reported reference velocities, experimental results are significantly lower than in ESDU 85020 (1985), in agreement with trends reported by Farell and Iyengar (1999).

Results reported in this section indicate that boundary layer wind tunnels are not capable of fully reproducing all features of atmospheric turbulence, in particular dependence of average eddy size on

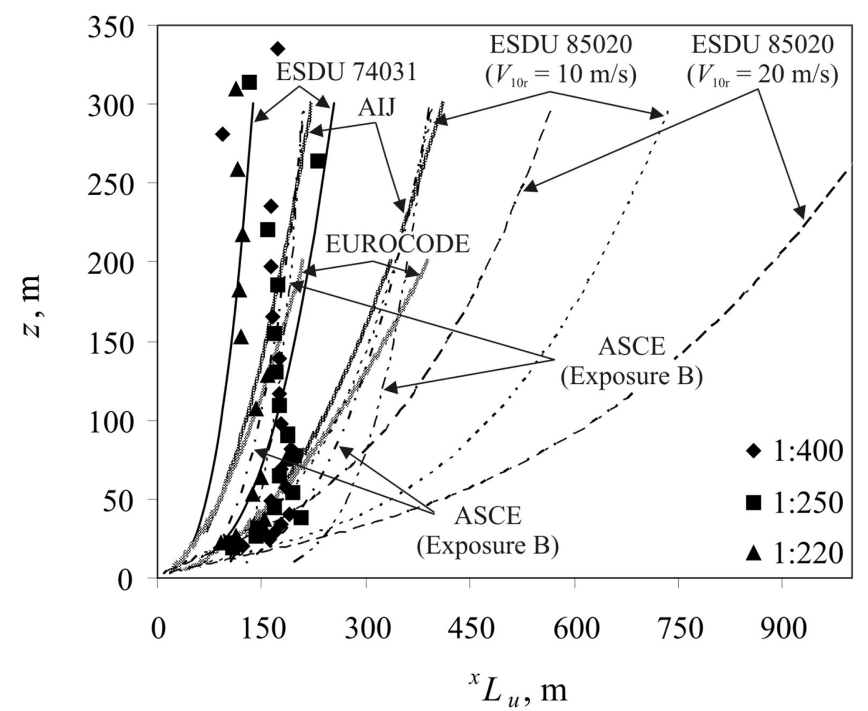

Fig. 16 Profiles of the integral length scales of turbulence ${ }^{x} L_{u}$ in the 1:400, 1:250 and 1:220 suburban ABL wind-tunnel simulations compared to values recommended in international standards 
reference wind velocity. Furthermore, for the suburban type terrain recommended integral length scales in ESDU 85020 (1985) are significantly larger than in other international standards, while ESDU 85020 (1985) recommended turbulence intensities compare reasonably with other standards, especially for smaller reference wind velocities.

\section{Conclusions}

Characteristics of the atmospheric boundary layer flow developing above suburban type terrain were physically reproduced in a boundary layer wind-tunnel for simulation length scale factors 1:400, 1:250 and 1:220. These length scale factors calculated using the Cook's (1978) procedure were justified through correspondence of boundary layer parameters in the wind tunnel and in the full-scale. Both mean winds and atmospheric turbulence were studied, and experimental results were compared to recommended values in international standards and codes. The main conclusions resulting from the present work can be summarised as follows:

- It is possible to generate suburban ABL wind-tunnel simulations at different simulation length scales (e.g., in this study 1:400, 1:250 and 1:220) without a significant influence of the simulation length scale factor on airflow characteristics, i.e., suburban ABL wind-tunnel simulations generated at different simulation length scales can have very similar characteristics.

- In the wind tunnel it is not possible to fully reproduce all features of atmospheric turbulence, in particular dependence of average eddy size on reference wind velocity indicated in ESDU 85020 (1985) and a linear increase in integral length scales of turbulence with height reported in Arya (1999).

- In international wind loading standards and codes there is a considerable scatter of recommended values for suburban wind characteristics. In particular, recommended integral length scales in ESDU 85020 (1985) are significantly larger than in other international standards. Turbulence intensities recommended in ESDU 85020 (1985) compare reasonably with other standards, especially for smaller reference wind velocities.

- Truncated vortex generators were successful in part-depth suburban ABL wind-tunnel simulation. However, further research is required to investigate a full range of their applicability.

\section{Acknowledgements}

This study was carried out at the Institute of Aerodynamics, Faculty of Mechanical Engineering, Technische Universität München (TUM). The support of the Croatian Ministry of Science and Technology, the German Academic Exchange Service (DAAD) and the Croatian Academy of Sciences and Arts (HAZU) are gratefully acknowledged. The author acknowledges helpful discussions with Prof. Boris Laschka, Dr. Albert Pernpeintner and Dr. Joseph Fischer. Special thanks needs to be expressed to the TUM departmental technical staff for the manufacturing of the simulation hardware.

\section{References}

AIJ (1996), AIJ recommendations for loads on buildings (in English), Architectural Institute of Japan, Tokyo. 
American Society of Civil Engineers (ASCE) (2006), Minimum design loads for buildings and other structures, ASCE/SEI 7-05, Reston, Virginia, USA.

Arya, S.P. (1999), Air pollution meteorology and dispersion, Oxford University Press, USA.

Arya, S.P. (2001), Introduction to Micrometeorology, 2nd Ed., Academic Press, New York.

Balendra, T., Shah, D.A., Tey, K.L. and Kong, S.K. (2002), "Evaluation of flow characteristics in the NUS-HDB wind tunnel", J. Wind Eng. Ind. Aerod., 90(6), 675-688.

Bendat, J.S. and Piersol, A.G. (2000), Random data: analysis and measurement procedures, John Wiley \& Sons, New York.

Blocken, B., Stathopoulos, T. and Carmeliet, J. (2007), "CFD simulation of the atmospheric boundary layer: wall function problems", Atmos. Environ., 41(2), 238-252.

Blocken, B., Stathopoulos, T., Saathoff, P. and Wang, X. (2008), "Numerical evaluation of pollutant dispersion in the built environment: Comparisons between models and experiments", J. Wind Eng. Ind. Aerod., 96(10-11), 1817-1831.

Cermak, J.E., Cochran, L.S. and Leffler, R.D. (1995), "Wind-tunnel modeling of the atmospheric surface-layer", J. Wind Eng. Ind. Aerod., 54, 505-513.

Cook, N.J. (1978), "Determination of the model scale factor in wind-tunnel simulations of the adiabatic atmospheric boundary layer", J. Wind Eng. Ind. Aerod., 2(4), 311-321.

Counihan, J. (1969a), "A method of simulating a neutral atmospheric boundary layer in a wind tunnel", Proceedings of the Advisory Group for Aerospace Research and Development (AGARD) Conference.

Counihan, J. (1969b), "An improved method of simulating an atmospheric boundary layer in a wind tunnel", Atmos. Environ., 3(2), 197-214.

Counihan, J. (1971), "Wind tunnel determination of roughness length as a function of fetch and roughness density of 3-dimensional roughness elements", Atmos. Environ., 5(8), 637-642.

Counihan, J. (1973), "Simulation of an adiabatic urban boundary-layer in a wind-tunnel", Atmos. Environ., 7(7), 673-689.

Counihan, J. (1975), "Adiabatic atmospheric boundary layers: a review and analysis of data from the period 1880-1972", Atmos. Environ., 9(10), 871-905.

Dyrbye, C. and Hansen, S.O. (1997), Wind loads on structures, John Wiley \& Sons, New York.

ESDU 72026 (1972), Characteristics of wind speed in the lower layers of the atmosphere near the ground: strong winds (neutral atmosphere), Engineering Sciences Data Unit.

ESDU 74031 (1974), Characteristics of atmospheric turbulence near the ground, Part II: Single point data for strong winds (neutral atmosphere), Engineering Sciences Data Unit.

ESDU 85020 (1985), Characteristics of atmospheric turbulence near the ground: Part II: single point data for strong winds (neutral atmosphere), Engineering Sciences Data Unit.

EN 1991 Eurocode 1 (2005), Actions on structures - General actions - Part 1-4: Wind actions.

Fang, C. and Sill, B.L. (1992), "Aerodynamic roughness length - correlation with roughness elements", J. Wind Eng. Ind. Aerod., 41(1-3), 449-460.

Farell, C. and Iyengar, A.K.S. (1999), "Experiments on the wind tunnel simulation of atmospheric boundary layers", J. Wind Eng. Ind. Aerod., 79(1-2), 11-35.

Flay, R.G.J. and Stevenson, D.C. (1988), "Integral length scales in strong winds below 20-m", J. Wind. Eng. Ind. Aerod., 28(1-3), 21-30.

Gartshore, I.S. and De Croos, K.A. (1977), "Roughness element geometry required for wind tunnel simulations of the atmospheric wind", J. Fluid Eng.-T. ASME, 99(3), 480-485.

Hargreaves, D.M. and Wright, N.G. (2007), "On the use of the k-epsilon model in commercial CFD software to model the neutral atmospheric boundary layer", J. Wind Eng. Ind. Aerod., 95(5), 355-369.

Harris, R.I. (1986), "Longer turbulence length scales", J. Wind Eng. Ind. Aerod., 24(1), 61-68.

Holmes, J.D. (2007), Wind loading of structures, 2nd Ed., Routledge, Taylor \& Francis, UK.

Holmes, J.D., Baker, C.J., English, E.C. and Choi, E.C.C. (2005), "Wind structure and codification", Wind Struct., 8(4), 235-250.

Hucho, W.H. (2002), Aerodynamik der stumpfen Körper, Vieweg \& Sohn, Wiesbaden.

Hunt, A. (1982), "Wind-tunnel measurements of surface pressures on cubic building models at several scales", $J$. Wind Eng. Ind. Aerod., 10(2), 137-163. 
ISO 4354 (1997), Wind actions on structures, International Standard Organization.

Kolmogorov, A.N. (1941), "The local structure of turbulence in incompressible viscous fluid for very large Reynolds numbers", Proceedings of the USSR Academy of Sciences, 30, 299-303.

Kozmar, H. (2008), "Influence of spacing between buildings on wind characteristics above rural and suburban areas", Wind Struct., 11(5), 413-426.

Kozmar, H. (2009), "Natural wind simulation in the TUM boundary layer wind tunnel", Proceedings of the 5th European-African Conference on Wind Engineering, Florence, Italy, July.

Kozmar, H. (2010), "Scale effects in wind tunnel modeling of an urban atmospheric boundary layer", Theor. Appl. Climatol., 100(1-2), 153-162.

Kozmar, H., Džijan, I. and Šavar, M. (2005), "Uniformity of atmospheric boundary layer model in the wind tunnel (in Croatian)", Strojarstvo, 47(5-6), 157-167.

Lim, H.C., Castro, I.P. and Hoxey, R.P. (2007), "Bluff bodies in deep turbulent boundary layers: Reynoldsnumber issues", J. Fluid Mech., 571, 97-118.

Melbourne, W.H. (1979), "Turbulence effects on maximum surface pressures-a mechanism and possibility of reduction", Proceedings of the 5th International Conference on Wind Engineering, Fort Collins, CO, USA, July.

Pernpeintner, A., Schnabel, P., Schuler, A. and Theurer, W. (1995), Appendix 17: Qualifizierungsversuch, WTGMerkblatt über Windkanalversuche in der Gebäudeaerodynamik, (Ed. Plate E.J.), WTG-Berichte Nr. 3, Windtechnologische Gesellschaft WTG e.V.

Peterka, J.A., Hosoya, N., Dodge, S., Cochran, L. and Cermak, J.E. (1998), “Area-average peak pressures in a gable roof vortex region", J. Wind Eng. Ind. Aerod., 77-78(1), 205-215.

Plate, E.J. (1982), Wind tunnel modelling of wind effects in engineering, Engineering Meteorology, Elsevier Scientific Publishing Company, Amsterdam, New York.

Schlichting, H. and Gersten, K. (1997), Grenzschicht-Theorie, 9th Ed., Springer, Berlin.

Simiu, E. and Scanlan, R.H. (1996), Wind Effects on Structures, 3rd Ed., John Wiley \& Sons, New York.

Sockel, H. (1984), Aerodynamik der Bauwerke, Vieweg \& Sohn, Braunschweig.

Stathopoulos, T. and Surry, D. (1983), "Scale effects in wind-tunnel testing of low buildings", J. Wind Eng. Ind. Aerod., 13(1-3), 313-326.

Stull, R.B. (2003), An Introduction to Boundary Layer Meteorology, Kluwer Academic Publishers, Dordrecht.

Tamura, Y., Iwatani, Y., Hibi, K., Suda, K., Nakamura, O., Maruyama, T. and Ishibashi, R. (2007), "Profiles of mean wind speeds and vertical turbulence intensities measured at seashore and two inland sites using Doppler sodars", J. Wind Eng. Ind. Aerod., 95(6), 411-427.

Tieleman, H.W. (1990), "Wind-tunnel simulation of the turbulence in the surface-layer", J. Wind Eng. Ind. Aerod., 36, 1309-1318.

Tieleman, H.W. (2003), "Wind-tunnel simulation of wind loading on low-rise structures: a review", J. Wind Eng. Ind. Aerod., 91(12-15), 1627-1649.

Von Kármán, T. (1948), "Progress in the statistical theory of turbulence", Proc. Natl. Acad. Sci. USA, 34(11), 530-539.

Wang, Z.Y., Plate, E.J., Rau, M. and Keiser, R. (1996), "Scale effects in wind tunnel modelling", J. Wind Eng. Ind. Aerod., 61(2-3), 113-130.

Yang, Y., Gu, M., Chen, S.Q. and Jin, X.Y. (2009), "New inflow boundary conditions for modelling the neutral equilibrium atmospheric boundary layer in computational wind engineering", J. Wind Eng. Ind. Aerod., 97(2), 88-95.

$J H$

\section{Notation}
$d$
displacement height
$d_{\mathrm{m}}$
displacement height in the wind tunnel 


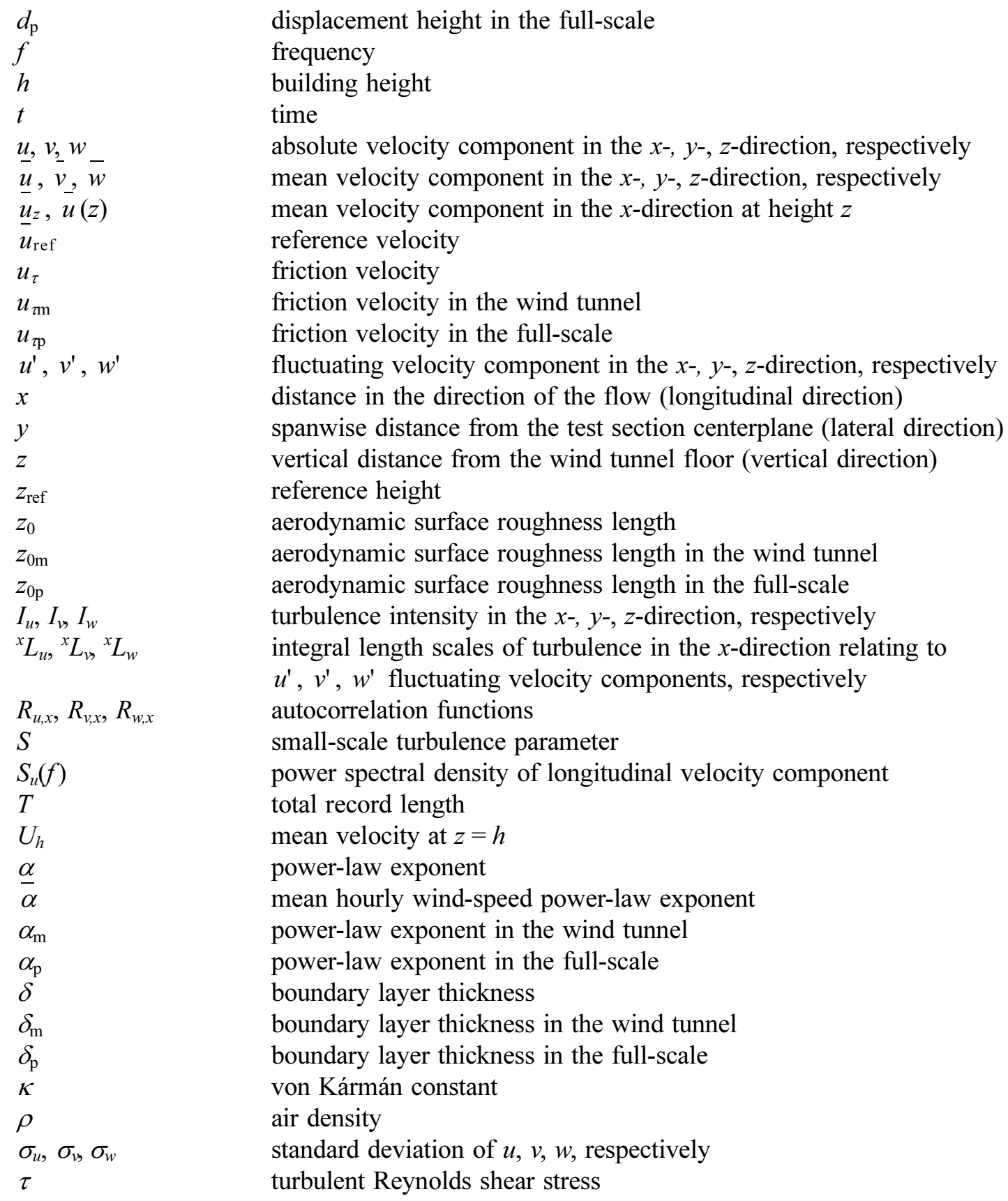

